\title{
Extended extraocular phenotype of PROM1 mutation in kindreds with known autosomal dominant macular dystrophy
}

\begin{abstract}
Francesca I Arrigoni ${ }^{1,2,10}$, Mar Matarin ${ }^{3,4,10}$, Pamela J Thompson ${ }^{3,4}$, Michel Michaelides ${ }^{5,6}$, Michelle E McClements ${ }^{5}$, Elizabeth Redmond ${ }^{1}$, Lindsey Clarke ${ }^{1}$, Elizabeth Ellins ${ }^{1}$, Saifullah Mohamed ${ }^{3,4}$, Ian Pavord ${ }^{7}$, David M Hunt ${ }^{5}$, Anthony T Moore ${ }^{5,6}$, Julian Halcox ${ }^{1,9}$ and Sanjay M Sisodiya ${ }^{\star, 3,4}$

Mutations in prominin 1 (PROM1) have been shown to result in retinitis pigmentosa, macular degeneration and cone-rod dystrophy. Because of the putative role of PROM1 in hippocampal neurogenesis, we examined two kindreds with the same R373C PROM1 missense mutation using our established paradigm to study brain structure and function. As the protein encoded by PROM1, known as CD133, is used to identify stem/progenitor cells that can be found in peripheral blood and reflect endothelial reparatory mechanisms, other parameters were subsequently examined that included measures of vascular function, endothelial function and angiogenic capacity. We found that aspects of endothelial function assayed ex vivo were abnormal in patients with the R373C PROM1 mutation, with impaired adhesion capacity and higher levels of cellular damage. We also noted renal infections, haematuria and recurrent miscarriages possibly reflecting consequences of abnormal tubular modelling. Further studies are needed to confirm these findings.
\end{abstract}

European Journal of Human Genetics (2011) 19, 131-137; doi:10.1038/ejhg.2010.147; published online 22 September 2010

Keywords: PROM1; CD133; endothelial; tubule; sella turcica

\section{INTRODUCTION}

The effects of a single human gene mutation may be widespread and, in their breadth, often unsuspected. We have previously explored the cerebral structural and functional effects of mutations in genes expressed in the eye and the brain, in individuals ascertained by their mutant eye phenotype, in whom extraocular phenotypes were often not appreciated. Using this paradigm, we have demonstrated roles for the genes PAX6, PITX2, SOX2, OTX2 and RIMS1 in human brain development and cognitive function. ${ }^{1-4}$

Mutations in PROM1 have been shown to result in retinitis pigmentosa, ${ }^{5,6}$ macular degeneration ${ }^{7,8}$ and cone-rod dystrophy. ${ }^{9}$ PROM1 encodes prominin-1, a 5-transmembrane glycoprotein also known as CD133 and AC133. CD133 was originally identified as a cell surface antigen present on hematopoietic stem cells and on early progenitor cells in the bone marrow, including endothelial progenitor cells (EPCs). ${ }^{10,11}$

Emerging evidence suggests that EPCs are able to differentiate into mature endothelial cells, contributing to neovascularization and reendothelialization during both embryonic and postnatal physiological processes. ${ }^{12}$ Consequently, CD133 is used to identify stem/progenitor cells that can be found in peripheral blood and thereby identify endothelial reparatory mechanisms and also to identify tumour stem cell populations in a variety of blood and solid cancers. ${ }^{13,14}$
Consistent with a role in tissue repair, increased levels of circulating early EPCs marked by CD133 have also been found in response to brain injury. ${ }^{15} \mathrm{CD} 133$ is also involved in murine and human adult hippocampal neurogenesis, ${ }^{16}$ murine brain myelin genesis and maintenance. ${ }^{17}$

Because of the putative role of PROM1 in hippocampal neurogenesis, we examined two kindreds with the same known PROM1 mutation using our established paradigm to study brain structure and function. We subsequently examined other parameters including measures of vascular function, endothelial function and angiogenic capacity.

\section{METHODS}

The study included two unrelated kindreds that were already reported as having autosomal dominant macular degeneration mapped to 4p15.2-16.3.5.18 From kindred A, the proband (A-V:1), her mother (A-IV:2), aunt (A-IV:4) and grandmother (A-III:6) were studied. In kindred B, the proband (B-II:1) and her mother (B-I:1) were included in the study (Table 1). In both probands, the same R373C PROM1 mutation had been identified. Clinical history was obtained from all participants.

Healthy control groups were recruited from staff and colleagues from the Institute of Child Health.

The study was approved by the Joint Research Ethics Committees of the Institute of Neurology/National Hospital for Neurology and Neurosurgery, and

\footnotetext{
${ }^{1}$ Department of Vascular Physiology, UCL Institute of Child Health, London, UK; ${ }^{2}$ Department of Pharmacy, Kingston University, Surrey, UK; ${ }^{3}$ Department of Clinical and Experimental Epilepsy, UCL Institute of Neurology, Queen Square, London, UK; ${ }^{4}$ National Society for Epilepsy, Chalfont-St-Peter, Bucks, UK; ${ }^{5}$ UCL Institute of Ophthalmology, London, UK; ${ }^{6}$ Moorfields Eye Hospital, London, UK; ${ }^{7}$ Department of Respiratory Medicine, Allergy and Thoracic Surgery, Institute for Lung Health, Glenfield Hospital, University Hospitals of Leicester NHS Trust, Leicester, UK; ${ }^{8}$ Research Department of Infection, UCL Institute of Child Health, London, UK and ${ }^{9}$ Wales Heart Research Institute, Cardiff University, Cardiff, UK

*Correspondence: Professor SM Sisodiya, Department of Clinical and Experimental Epilepsy, UCL, National Hospital for Neurology and Neurosurgery, Box 29, Queen Square, London WC1N 3BG, UK. Tel: +44 207391 8983; Fax: +44 207391 8984; E-mail: sisodiya@ion.ucl.ac.uk

10These authors contributed equally to this work.

Received 18 January 2010; revised 8 July 2010; accepted 22 July 2010; published online 22 September 2010
} 
Table 1 Summary of age-corrected cognitive performance (in italics poor or impaired scores) and extraocular findings

\begin{tabular}{|c|c|c|c|c|c|c|}
\hline \multirow{2}{*}{$\begin{array}{l}\text { R373C PROM1 mutation } \\
\text { Subject }\end{array}$} & \multicolumn{4}{|c|}{ Family $A$} & \multicolumn{2}{|c|}{ Family B } \\
\hline & $A-I I I: 6$ & $A-I V: 4$ & $A-I V: 2$ & $A-V: 1$ & $B-I: 1$ & $B-I I: 1$ \\
\hline Relationship to proband & Maternal grandmother & Maternal aunt & Mother & Proband & Mother & Daughter \\
\hline $\mathrm{VIQ}$ & 114 & 90 & 96 & 110 & 106 & 110 \\
\hline Verbal learning trials & 46 (50th) & 46 (25th) & 51 (50th) & 50 (25th) & $22(<1 s t)$ & 56 (50th) \\
\hline Verbal learning delay & 7 (25th) & 9 (10th) & 13 (75th) & 12 (50th) & 6 (10th) & 14 (75th) \\
\hline Fluency phonemic 's' & 12 & 19 & 16 & 18 & 15 & 24 \\
\hline Fluency animals & 19 & 15 & 26 & 24 & 20 & 28 \\
\hline Hayling's total score & 14 & 16 & 13 & 18 & 11 & 17 \\
\hline Cognitive estimates & 5 & 12 & 8 & 7 & 4 & 5 \\
\hline Extraocular medical history & $\mathrm{RI}, \mathrm{M}, \mathrm{MC}$ & M & $\mathrm{MH}, \mathrm{RI}, \mathrm{MC}$ & SRA, MH, NE & Normal & M \\
\hline MRI findings & WMH, EST & WMH, EST & EST & Normal & WMH & Normal \\
\hline
\end{tabular}

Abbreviations: EST, empty sella turcica; EX, examined; M, microsmia; MC, miscarriages; $\mathrm{MH}$, microscopic haematuria; NE, not examined; RI, renal infections; SRA, steroid-resistant asthma; VIQ, verbal intelligence quotient; WMH, white matter hyperintensities.

Moorfields Eye Hospital and the Institute of Child Health/Great Ormond Street Hospital for Children NHS Foundation Trust. Subjects provided informed written consent for all parts of the study.

\section{Brain imaging}

Subjects were studied on a 3T scanner. Details of the high-resolution MRI, cerebral and hippocampal volumetry are provided in the Supplementary Material.

\section{Cognitive tests applied to kindreds}

Neuropsychological tests employed were as described previously. ${ }^{19}$ All of the standardised tests selected require verbal interaction only. Intellectual level, executive and memory function were tested. Details are given in the Supplementary Material.

\section{Clinical testing}

Proband A-V:1 had renal and pulmonary assessments previously undertaken owing to relevant clinical symptoms. Olfactory testing was undertaken using the UPSIT ${ }^{1}$ (Sensonics Inc, Haddon Heights, NJ, USA), with age-matched control data based upon North American subjects, shown to be applicable to British populations. ${ }^{20,21}$

\section{Blood and vascular investigations}

After a 12-h overnight fast, venous blood was taken from three subjects from kindred A and one from kindred B. After subjects had rested for $>15 \mathrm{~min}$, recumbent right brachial artery blood pressure was recorded using an automated sphygmomanometer (Dinamap, Critikon, FL, USA). Five readings were taken, and mean systolic and diastolic blood pressure was calculated.

The isolation of peripheral blood mononuclear cells, flow cytometric analysis for detection of EPCs and cellular microparticles (MPs), circulating endothelial cell (CEC) extraction and analyses, growth factor detection, the ability of specific cells to form colonies, microparticle assays and in vitro colony-forming and Matrigel angiogenesis assays were performed as detailed in the Supplementary Material. For kindred A, vascular structure (carotid artery intima-media thickness, IMT) and function (flow-mediated dilatation (FMD) of brachial artery) were also assessed (Supplementary Material).

\section{Statistics}

Non-parametric tests (Mann-Whitney $U$ ) were used with SPSS, version 14 (SPSS Inc., Chicago, IL, USA). Data were expressed as median and interquartile range.

\section{RESULTS}

\section{Clinical findings}

The ocular phenotypic data have been published. ${ }^{5,18}$ A-V:1 had steroid-resistant asthma. She had a clinical assessment for microscopic haematuria, with a normal renal ultrasound and intravenous pyelogram. A-IV:2 also had microscopic haematuria, with recurrent renal infection and renal scarring on intravenous pyelography. She had eight pregnancies and four children. A-III:6 had recurrent renal infections, seven pregnancies with three miscarriages and four children. A-IV:4 had two unprovoked seizures.

In kindred B, B-I:1 had high blood pressure and high cholesterol.

According to North American normative data, UPSIT A-IV:4 and B-II:1 had mild microsmia and A-III:6 had moderate microsmia.

\section{Neuroimaging}

Subject A-III:6 had many small high-intensity lesions in the cerebral white matter (WM; Figure 1b). Subjects A-IV:4 and B-I:1 also had a few (1-2) non-specific WM lesions. The presence of WM hyperintensities on the FLAIR images for subject A-III:6 was scored by a radiologist blind to the clinical data, using the Scale of TarvonenSchroder and Scheltens semiquantative rating scale. ${ }^{22}$ This scale scores the severity of hyperintensities separately in different anatomical areas of the brain, including periventricular regions, areas of deep WM, basal ganglia and different infratentorial regions. The total score was 19. Lesions were focused on deep WM (score $=16 / 24)$ and pons $($ score $=3 / 6)$. Lesions in frontal brain area were considered as severe and mild-to-moderate in the other brain areas.

An empty sella turcica was noted in A-III:6, A-IV:2 and A-IV:4 (Figure 1a). Baseline pituitary function (TSH, T4, cortisol, FSH and LH) was normal in all three. No other changes were noted. MRI brain scans in kindred B were normal. Hippocampal and cerebral volumes were within normal ranges for all patients from both kindreds.

\section{Cognitive function}

No distinctive pattern of cognitive deficit was noted (Table 1). All subjects in kindred A except A-IV:4 showed average to superior verbal memory scores. A-IV:4 showed poor performance for delayed recall on 

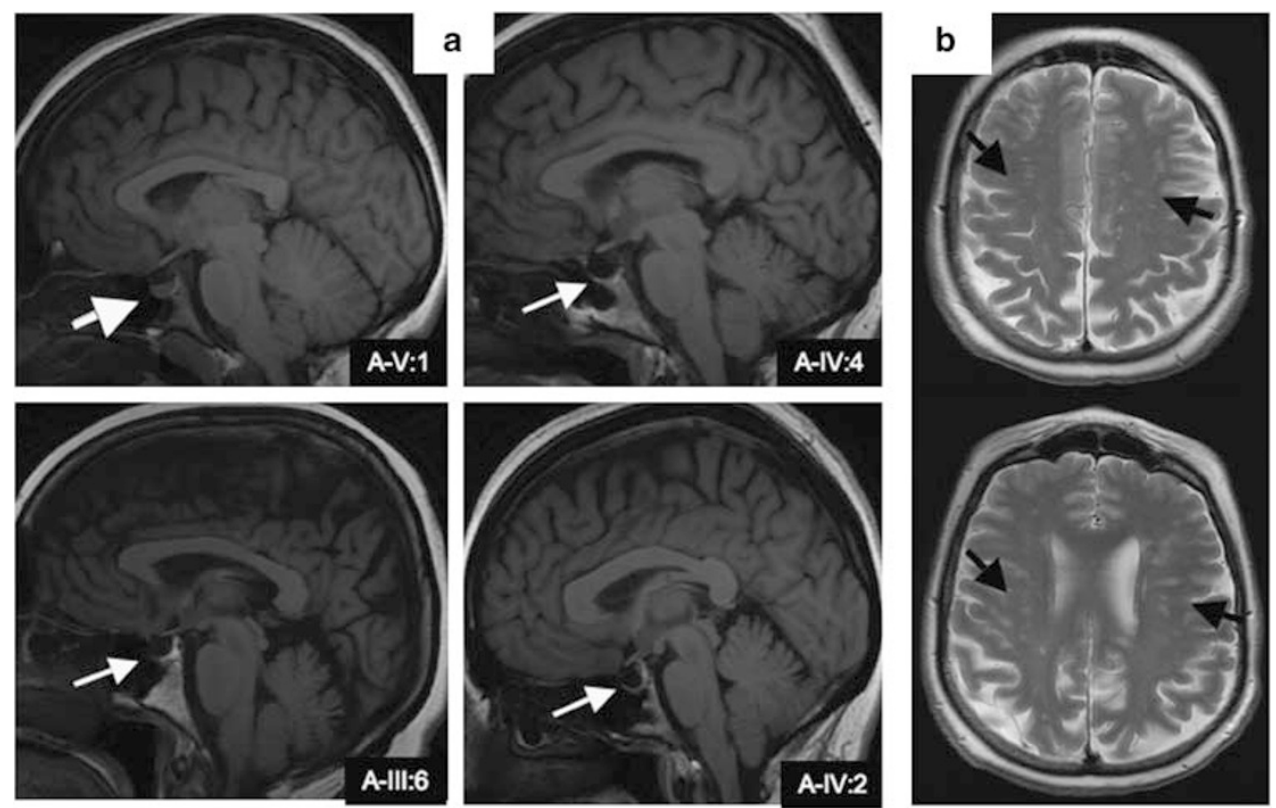

Figure 1 (a) The white arrows show empty sella turcica in three PROM1 patients (A-IV:4, A-III:6 and A-IV:2) and one normal sella turcica (A-V:1). (b) The black arrows show WM hyperintensities in one PROM1 patient (A-III:6).

Table 2 Blood/vasculature tests in controls and PROM1 subjects

\begin{tabular}{|c|c|c|c|c|c|c|c|c|}
\hline \multirow{2}{*}{$\begin{array}{l}\text { Age group } \\
\text { ID }\end{array}$} & \multicolumn{3}{|c|}{1} & \multicolumn{2}{|r|}{2} & \multicolumn{2}{|r|}{3} & \multirow[t]{2}{*}{ P-values } \\
\hline & $A-V: 1$ & $B-I I: 1$ & Control & $A-I V: 2$ & Control & $A-I I I: 6$ & Control & \\
\hline Age & 19 & 32 & $21(17-24)$ & 46 & $46(45-47)$ & 66 & $55(53-61)$ & \\
\hline Percentage of FMD & 12.27 & & $7.4(4.5-11.4)$ & 4.06 & $9.4(6.1-12)$ & 4.88 & $7.27(6.2-11.5)$ & NS \\
\hline FMD/NTG & 1.02 & & $0.95(0.9-1)$ & 0.98 & $0.9(0.9-1)$ & 0.96 & $0.95(0.9-1)$ & NS \\
\hline Average IMT (mm) & 0.48 & & $0.58(0.6-0.6)$ & 0.52 & $0.64^{\#}(0.6-0.7)$ & 0.75 & $0.76(0.7-0.8)$ & NS $\left[{ }^{* \#}\right]$ \\
\hline CEC (ml blood) & 28 & 16 & $32(15-38)$ & 56 & $64(52-78)$ & 48 & 74 (27-108) & NS [0.04] \\
\hline $\operatorname{CD~} 133^{+}$ & 0.05 & 0.25 & $0.05(0-0.03)$ & 0 & $0.02(0.02-0.46)$ & 0.03 & $0.025(0.02-0.6)$ & NS \\
\hline $\mathrm{CD} 34^{+}$ & 0.01 & 0.17 & $0.13(0.06-0.39)$ & 0.2 & $0.2(0.12-0.37)$ & 0.2 & $0.25(0.05-0.63)$ & NS \\
\hline CD $144^{+}$ & 0.76 & & $0.53(0.44-1.71)$ & 1.34 & $0.39(0.13-1.89)$ & 4.69 & $1.05(0.81-2.61)$ & NS \\
\hline $\mathrm{KDR} / \mathrm{CD} 34^{+}$ & 0.06 & 0.07 & $0.07(0.06-0.2)$ & 0.02 & $0.05(0.02-0.08)$ & 0.05 & $0.05(0.03-0.1)$ & NS \\
\hline CD144/CD34+ & 0.03 & 0.02 & $0.03(0.01-0.21)$ & 0.08 & $0.025(0.01-0.05)$ & 0.09 & $0.025(0.01-0.05)$ & NS \\
\hline
\end{tabular}

Abbreviations: NS, not significant; FMD, flow-mediated dilatation; GTN, glyceryl trinitrate (25 $\mu$ g); CEC, circulating endothelial cell; IMT, intima-media thickness; VEGF, vascular endothelial growth factor. Values represent median (interquartile range); $P$-values=statistics are for comparisons between patients and controls. ${ }^{*} P=0.0008$ compared with group 1 . ${ }^{\#} P=0.008$ compared with group 3.

${ }^{+}=$Surface markers. []$=$Comparisons within age control groups.

the auditory verbal learning test with an average performance on the remaining tests of memory. In kindred B, B-I:1 had impaired verbal learning, for trials reduced performance on tests of immediate recall and delayed verbal learning. All subjects except A-IV:4 and B-I:1 showed average executive functions.

\section{Vascular and endothelial function}

Vascular profiles. Subjects A-V:1, A-IV:2 and A-III:6 underwent vascular investigation. Each subject was ranked into an age group, (1, 2 or 3$)$, in which 12 healthy controls, with a median age similar to that of the subject, were included (see Table 2). Patients and controls were all females and had no known cardiovascular risk factors, with the exception of high blood pressure and high cholesterol in subject B-1:1.
Brachial artery FMD and carotid artery IMT. Detailed descriptions of procedures are in Supplementary Material. Briefly, the diameter of the brachial artery was measured at rest, during reactive hyperaemia to induce endothelium-dependent FMD, again at rest and after administration of glyceryl trinitrate (GTN), an endothelium-independent dilator. FMD and the ratio FMD:GTN-induced dilatation provide the best measures of endothelial function.

FMD did not alter with age in the controls (Table 1). There was no difference in median FMD when comparing grouped controls and patients (8.28 (5.68-11.40) and 4.88 (4.06-12.27)). The ratio FMD:GTN did not alter with age in controls, but fell with age in the patients. The median FMD:GTN ratio for the grouped patients did not differ from the controls $(0.95(0.90-0.97)$ and $0.98(0.96-1.02))$. 
IMT increased significantly with age in the controls (Table 2). Increasing IMT values were also observed in the patients. However, there was no difference in the median values for IMT between grouped controls and PROM1 patients $(0.65(0.58-0.72)$ and $0.52(0.48-0.75))$.

Determination of growth factor concentration. Angiopoietin-2 levels and vascular endothelial growth factor levels were within the normal range according to established clinical ranges.

Isolation and quantification of EPCs. In controls, CEC number significantly increased between the younger groups 1 and 2, but not between either of the other age groups (Table 2). Increase in CECs with age is less evident in PROM1 patients but falls within ranges for healthy age-matched controls (Table 2). No change in the expression of surface markers was observed with age in controls or PROM1 patients. Calculated as a percentage of the lymphocyte gate, there was no significant difference between groups in the expression of any of the surface markers alone or in combination (Table 2).

Cultivation of EPCs. Colony-forming unit endothelial cells (CFU-EC) fell with age in controls. This trend was also observed in the few PROM1 patients. Colony number tended to be low, but values fell within the normal range for their ages (controls, 20.37 (7.05-32.57) and PROM1 patients, 11.9 (4.6-41)).

Relationship between cellular and vascular measures. Numbers of CFU-EC correlated with FMD:GTN ratio in the controls $(r=0.42$, $P<0.05)$. CFU-EC from PROM1 patients demonstrated a similar trend.

Microparticle number. Total microparticle number, which consisted of endothelial MPs (CD144, D105 and CD62e), platelet MPs (CD42a) and monocyte-derived MPs (CD14), fell within the upper range in PROM1 patients in relation to age-matched controls. Comparisons between patients and controls independently to age showed higher number of total MPs in PROM1 patients (2.606 (1.399-2.97) and $0.3023(0.14-1.35)$ for PROM1 patients and healthy age-matched controls, respectively, median \pm interquartile range, $P=0.0028$ ). Furthermore endothelial MPs defined by CD144 expression were also found to be significantly elevated in the PROM1 progeny (Table 3).

Angiogenic capability. A Matrigel assay was used to determine the effect of CFU-ECs labelled with acetylated low-density lipoprotein (DiI-acLDL) on human umbilical endothelial cell (HUVEC) growth.

DiI-acLDL-labelled cells from PROM1 patients did not adhere to or incorporate into the HUVECs as well as their age-matched controls
(5.6 (0-18) and 26.7 (15.5-44.3), $P=0.03$; Figure 2). Furthermore, a lack of Dil-acLDL-labelled cell incorporation into the HUVEC tubules tended to produce lower tubule length in PROM1 subjects when compared with their age-matched controls $(0.8(0.74-1.2)$ and 1.38 (0.9-1.46), $P=0.06$; Figures 2 and 3). No difference in tubule number was observed between the two groups (1 (1-1.14) and 0.88 (0.52-1.22), $P=0.57$; Figure 3).

\section{DISCUSSION}

We describe vascular and endothelial function in addition to cerebral structure and function in two independent families with the same R373C mutation in the PROM1 gene. Carriers of the mutation were already reported to present clinical features of autosomal dominant macular dystrophy. ${ }^{7,18}$

Human PROM1 is expressed by various stem and progenitor cells originating from diverse sources. ${ }^{23}$ Owing to the role of EPCs in vascular and endothelial function, ${ }^{24}$ we investigated these characteristics in five carriers of the R373C mutation. We found that the number of CECs, endothelial markers such as CD133, as well as the ability of EPCs to form endothelial colonies fell within the normal range in the tested patients. Endothelium-dependent and -independent functions, assessed by FMD, and the FMD:GTN ratio did not differ from controls. There was also no difference in IMT. The basic function and EPC regenerative resources would seem unaffected by the R373C mutation.

However, the number of circulating MPs was raised in patients compared with controls. MPs are membrane fragments shed by cells which have been activated by a variety of stimuli including serine proteases, inflammatory cytokines, growth factors and stress inducers. ${ }^{25}$ Clinical studies have revealed elevated plasma levels of MPs in multiple sclerosis, thrombotic thrombocytopenic purpura, coronary artery disease, hypertension, preeclampsia and diabetes. ${ }^{26,27}$ Such data have led to the concept that endothelial MPs are key factors at the crossroads between inflammation, coagulation, proteolysis and vascular repair. ${ }^{28}$ Elevated circulating levels of MPs in our patients could be an indicator of either platelet, endothelial or leukocyte activation and could reflect a persistent state of endothelial dysfunction.

We looked also at the contribution of immature ECFCs to tubule growth. A significantly decreased ability of these cells to incorporate or adhere to the HUVECs was observed in carriers of the mutation. As this assay evaluates multiple cellular processes involved in blood vessel growth, EPC functional activity would appear impaired by the mutation. In turn, vascularization might be affected in the patients, at least under certain circumstances, perhaps those requiring neogenesis or repair.

Table 3 Expression of microparticle number in platelet-poor plasma taken from PROM1 subjects

\begin{tabular}{|c|c|c|c|c|c|c|c|c|}
\hline \multirow{3}{*}{ Age group } & \multicolumn{8}{|c|}{ Million microparticles/ml blood } \\
\hline & \multicolumn{3}{|c|}{1} & \multicolumn{2}{|c|}{2} & \multicolumn{2}{|r|}{3} & \multirow[t]{2}{*}{ P-values } \\
\hline & $A-V: 1$ & $B-I I: 1$ & Control & $A-I V: 2$ & Control & $A-I I I: 6$ & Control & \\
\hline Age & 19 & 32 & $28(23-31)$ & 46 & $40(38-43)$ & 66 & $64(61-65)$ & \\
\hline CD62e & 0.015 & 0.556 & $0.009(0-1.08)$ & 0.086 & $0.005(0-0.05)$ & 0.08 & $0.14(0.01-0.02)$ & 0.0279 \\
\hline CD144 & 1.19 & 0.774 & $0.02(0-0.7)$ & 1.27 & $0.33(0.13-0.41)$ & 0.8 & $0.15(0.04-0.21)$ & 0.0018 \\
\hline CD105 & 0.7 & 0.085 & $0.04(0-0.71)$ & 0.16 & $0.06(0.02-0.41)$ & 0.39 & $0.035(0.02-0.07)$ & 0.0613 \\
\hline CD42 & 0.47 & 0.56 & $0.2(0-0.88)$ & 0.57 & $0.23(0.18-0.38)$ & 1.15 & $0.085(0.07-1.04)$ & 0.0115 \\
\hline CD14 & 2.7 & 0.7 & $0.29(0-1.3)$ & 1.98 & $0.17(0.03-0.34)$ & 2.87 & $0.076(0.06-0.94)$ & 0.0028 \\
\hline Total MP number & 2.89 & 1.09 & $0.28(0.04-1.35)$ & 2.32 & $0.51(0.18-0.55)$ & 3 & $0.19(0.17-0.67)$ & 0.0028 \\
\hline
\end{tabular}

Data are expressed as median (range) microparticles per $\mathrm{ml}$ of blood or plasma; $P=$ statistics are for comparisons between PROM1 patients and controls. Significant $P$-values are in italics (after Bonferroni's correction). 

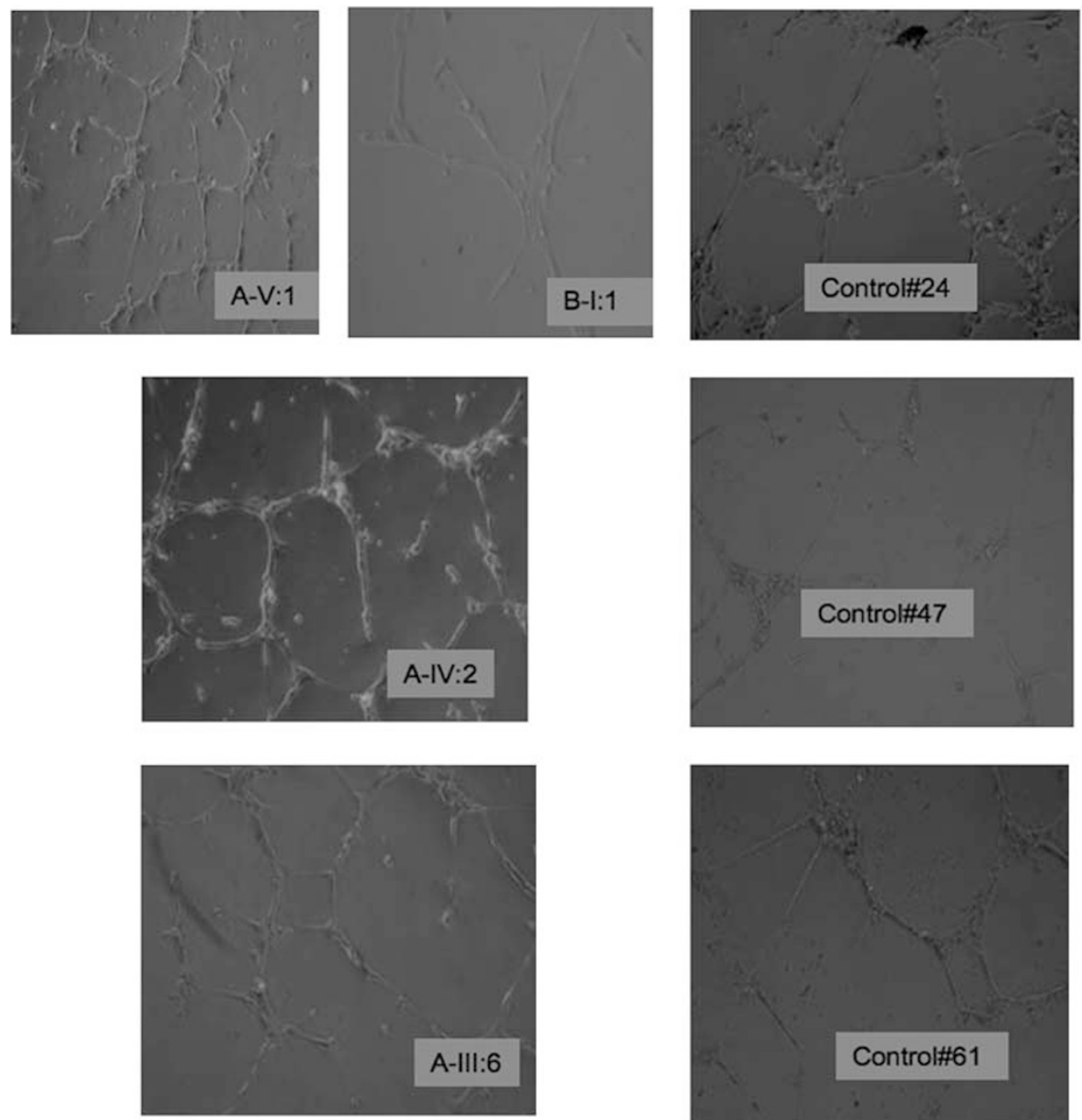

Figure 2 Representative micrograph of HUVECs in Matrigel for 18h with EGM-2 grown cells from PROM1 patients and age-matched controls (24, 47 and 61 years old). Cells labelled red are Dil-labelled EGM-2 grown cells after 7 days in culture. Fewer adherent (red-coloured) cells and a tendency for shorter tubule formation are apparent in patients compared with controls. The adhesion of the (red-labelled) ECFCs contributes to endothelial tubule formation. Following culture of ECFCs with HUVECs, fewer ECFCs were adherent or incorporated into the tubules from the PROM1 subject when compared with the age-matched control. Magnification $\times 100$, eight random fields studied in A-V:1, A-IV:2, A-III:6 and B-I:1.

a

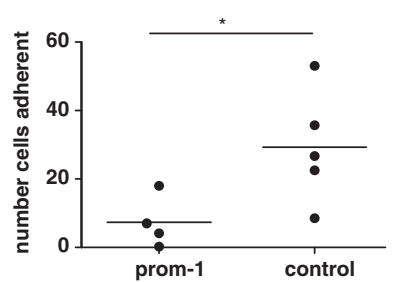

b

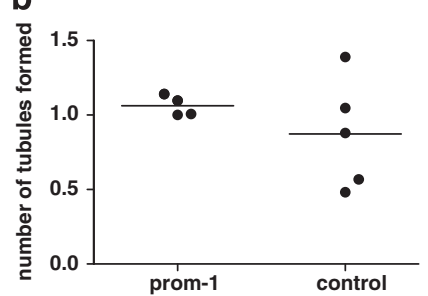

c

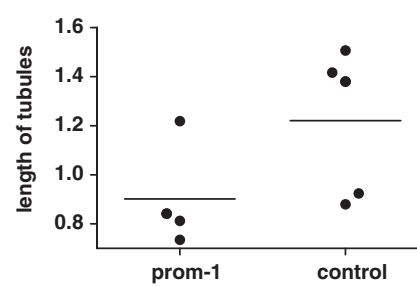

Figure 3 Angiogenesis assay for HUVECs and ECFCs. Alteration in growth of HUVEC tubules with ECFCs from PROM1 subjects. (a) Number of cells adherent/incorporated into endothelial tubules on Matrigel in PROM1 subjects and healthy age-matched controls; (b) Number of tubules formed on Matrigel plate, averaged over eight random fields between PROM1 patients and controls. (c) Average tubule length of HUVECs formed on Matrigel, averaged over eight random fields between PROM 1 and controls. ${ }^{*} P<0.05$ significant difference between PROM 1 and control group.

Notably, the mutation carriers had microhaematuria and/or renal infections and a high number of miscarriages. Although there is no study reporting endothelial dysfunction in patients with haematuria and/or renal infections, several studies have reported that EPCs are numerically and functionally impaired in patients with acute ${ }^{29}$ or chronic renal failure, ${ }^{30}$ as compared with healthy subjects, and mechanisms such as inflammation or uraemic toxins have been given as potential explanations for the observed EPC dysfunction. ${ }^{30}$ More clear and direct is the relationship between miscarriages and possible endothelial dysfunction, given that development, maturation 
and maintenance of a neovascular network are necessary for successful haemochorial placentation as well as normal embryonic development and growth. ${ }^{31}$ Impairment in EPC functionality could thus affect placental angiogenesis and contribute to history of recurrent miscarriages in our patients. This requires further study in a larger group of patients, and might merit study in women with unexplained recurrent miscarriages.

CD133 has been also reported to mark several cell types with a variety of putative roles, including involvement in hippocampal neurogenesis ${ }^{16,17}$ and diverse haematopoietic and extrahaematopoietic progenitive and regenerative functions. ${ }^{32-34}$ Carriers of the PROM1 mutation showed hippocampal and cerebral volumes within normal ranges for all patients from both kindreds. Three patients had small lesions in WM. Interestingly, the presence of MPs has also been reported to be positively associated with contrast-enhancing lesions on brain MRI in subjects with multiple sclerosis ${ }^{35}$ and furthermore, endothelial dysfunction is thought to have an important role in the pathogenesis of cerebral small-vessel disease especially in those patients with concomitant silent lacunar infarcts and ischaemic WM lesions. ${ }^{36}$ In line with this possible accelerated aging effect, the older member from the kindred $\mathrm{B}$ and one carrier of the PROM1 mutation in family A showed some memory disturbance and impairment in measures of executive functions in addition to small WM lesions. The remaining carriers in both families had average IQs and unimpaired performance on the other cognitive measures.

The three (of four) members of family A showed an empty sella turcica. The familial empty sella together with an eye phenotype could suggest a developmental defect of mesenchymal origin caused by mutation in the same gene. ${ }^{37}$ Familial empty sella has not, to our knowledge, been reported previously. Three patients had impaired olfaction.

All together, the elevated plasma levels of MPs, decreased ability of EPCs to incorporate into or adhere to tubules, the presence of empty sella turcica as well as leucoaraiosis and cognitive decline in the oldest mutation carriers suggest endothelial dysfunction that could be more pronounced or more evident with age. The $\mathrm{R} 373 \mathrm{C}$ mutation results in a stable mutant protein. However, the mutant protein is not only mislocalized in retina, but it also interferes with the action of the normal protein, ${ }^{7}$ thereby accounting for its dominant mode of inheritance. Though we found no difference in the number of circulating EPCs or numbers of cells expressing CD133, the mutation may impair the function. For example, the mutation could render EPCs less effective in the process of angiogenesis or repair of damaged endothelium. Carriers of the R373C mutation showed no obvious greater risk of vascular dysfunction, but structural and functional microcirculation alterations tend to occur during ageing. Functional vascular examination was only undertaken in one of the two older patients as the second declined to take part in this test. Moreover, the presence of high blood pressure and high cholesterol, as well as leucoaraiosis, ${ }^{38}$ could be a sign of vascular alteration. Longitudinal study of more individuals with PROM1 mutation might be informative.

To our knowledge, this is the first study reporting a family with eye phenotype together with empty sella turcica, presence of microhematuria and possible endothelial dysfunction. In the case of the eye phenotype (macular dystrophy), the penetrance was complete although showing differences in severity. Penetrance in the other phenotypes was incomplete. Notable is the fact that none of the members in family B presented extra-ocular features, nor MRI findings (other than a few WM lesions), besides the cognitive decline in the older member, raising the possibility of organ-specific penetrance differences, perhaps related to organ-specific splicing differences of the PROM1 transcript. PROM1 transcription regulation is rather complicated and poorly understood: it exhibits extensive splice variation ${ }^{39}$ with tissue-specific distribution, ${ }^{40}$ and in addition, transcripts can be regulated by epigenetic factors, as suggested by experiments with artificial in vitro methylation. ${ }^{40}$ However, we cannot exclude the possibility that mutation in a second gene could be influencing the extra-ocular phenotype in family A, especially the presence of empty sella turcica.

The role of PROM1 in brain is still unclear. The gene, by itself or in combination with other genes, seems to have a role in the development of the brain, and/or cerebral recovery and repair. ${ }^{17,41}$ The heterozygous mutation studied here results in a stable protein ${ }^{7}$ and is perhaps insufficient to completely inhibit its function in the brain. In contrast, recessive mutations arise from either frameshift or nonsense mutations ${ }^{5,6}$ that result in the generation of a premature stop codon and truncated protein. ${ }^{5,6,42}$ In these cases, the homozygous patients display a severe eye phenotype, and polydactyly was also reported in one of the carriers, an additional symptom that confirms the existence of variable penetrance associated with PROM1. No other documentation about vascular, endothelial or brain structures was reported.

The nature and size of our study does not permit definitive conclusions but leads to the suggestion that endothelial function may be affected in patients with the PROM1 R373C mutation, despite the apparently normal levels of EPCs. Further studies are needed to confirm these interesting findings that broaden the phenotype of PROM1 mutation, and inform our understanding of CD133 function.

\section{CONFLICT OF INTEREST}

The authors declare no conflict of interest.

\section{ACKNOWLEDGEMENTS}

We thank the patients who participated. We are grateful to the Big Lottery Fund, Wolfson Trust and the National Society for Epilepsy for supporting the NSE MRI scanner. The work was supported by a joint grant from the Biomedical Research Centres at UCLH/UCL, Great Ormond Street Hospital for Children/UCL Institute of Child Health and Moorfields Eye Hospital/UCL Institute of Ophthamology. This work was undertaken at UCLH/UCL, which received a proportion of funding from the Department of Health's NIHR Biomedical Research Centres funding scheme. Drs JE Halcox and FI Arrigoni were supported by the British Heart Foundation, Dr E Redmond by the Coronary Artery Disease Research Association, and Ms ME McClements by Fight for Sight.

1 Sisodiya SM, Free SL, Williamson KA et al: PAX6 haploinsufficiency causes cerebral malformation and olfactory dysfunction in humans. Nat Genet 2001; 28: 214-216.

2 Idrees F, Bloch-Zupan A, Free SL et al: A novel homeobox mutation in the PITX2 gene in a family with Axenfeld-Rieger syndrome associated with brain, ocular, and dental phenotypes. Am J Med Genet B Neuropsychiatr Genet 2006; 141B: 184-191.

3 Sisodiya SM, Ragge NK, Cavalleri GL et al: Role of SOX2 mutations in human hippocampal malformations and epilepsy. Epilepsia 2006; 47: 534-542.

4 Henderson RA, Williamson K, Cumming S et al: Inherited PAX6, NF1 and OTX2 mutations in a child with microphthalmia and aniridia. Eur J Hum Genet 2007; 15: 898-901.

5 Zhang Q, Zulfiqar F, Xiao X et al: Severe retinitis pigmentosa mapped to 4p15 and associated with a novel mutation in the PROM1 gene. Hum Genet 2007; 122: 293-299.

6 Maw MA, Corbeil D, Koch J et al: A frameshift mutation in prominin (mouse)-like 1 causes human retinal degeneration. Hum Mol Genet 2000; 9: 27-34.

7 Yang Z, Chen Y, Lillo $\mathrm{C}$ et al: Mutant prominin 1 found in patients with macular degeneration disrupts photoreceptor disk morphogenesis in mice.[see comment]. J Clin Investig 2008; 118: 2908-2916. 
8 Permanyer J, Navarro R, Friedman J et al: A novel mutation in PROM1 that results in nonsense mediated decay causes autosomal recessive retinitis pigmentosa with early macular affectation. Invest Ophthalmol Vis Sci 2010; 51: 2656-2663.

9 Michaelides M, Gaillard M, Escher P et al: The PROM1 mutation p.R373C causes an autosomal dominant bull's eye maculopathy associated with rod, rod-cone and macular dystrophy. Invest Ophthalmol Vis Sci 2010; 51: 4771-4780.

10 Yin AH, Miraglia S, Zanjani ED et al: AC133, a novel marker for human hematopoietic stem and progenitor cells. Blood 1997; 90: 5002-5012.

11 Miraglia S, Godfrey W, Yin AH et al: A novel five-transmembrane hematopoietic stem cell antigen: isolation, characterization, and molecular cloning. Blood 1997; 90: 5013-5021.

12 Urbich C, Dimmeler S: Endothelial progenitor cells: characterization and role in vascular biology. Circ Res 2004; 95: 343-353.

13 Singh SK, Hawkins C, Clarke ID et al: Identification of human brain tumour initiating cells.[see comment]. Nature 2004; 432: 396-401.

14 Zhu L, Gibson P, Currle DS et al: Prominin 1 marks intestinal stem cells that are susceptible to neoplastic transformation.[see comment]. Nature 2009; 457: 603-607.

$15 \mathrm{Liu} \mathrm{L,} \mathrm{Liu} \mathrm{H,} \mathrm{Jiao} \mathrm{J} \mathrm{et} \mathrm{al:} \mathrm{Changes} \mathrm{in} \mathrm{circulating} \mathrm{human} \mathrm{endothelial} \mathrm{progenitor} \mathrm{cells}$ after brain injury.[erratum appears in J Neurotrauma. 2007 Aug;24(8):1415 Note: Jao, Junfeng [corrected to Jiao, Junfeng]. J Neurotrauma 2007; 24: 936-943.

16 Kempermann G, Chesler EJ, Lu L et al: Natural variation and genetic covariance in adult hippocampal neurogenesis. Proc Natl Acad Sci USA 2006; 103: 780-785.

17 Corbeil D, Joester A, Fargeas CA et al: Expression of distinct splice variants of the stem cell marker prominin-1 (CD133) in glial cells. GLIA 2009; 57: 860-874.

18 Michaelides M, Johnson S, Poulson A et al: An autosomal dominant bull's-eye macular dystrophy (MCDR2) that maps to the short arm of chromosome 4. Invest Ophthalmol Vis Sci 2003; 44: 1657-1662.

19 Sisodiya SM, Thompson PJ, Need A et al: Genetic enhancement of cognition in a kindred with cone-rod dystrophy due to RIMS1 mutation. J Med Genet 2007; 44: 373-380.

20 Gibberd FB, Feher MD, Sidey MC et al: Smell testing: an additional tool for identification of adult Refsum's disease. J Neurol Neurosurg Psychiatry 2004; 75: 1334-1336.

21 Silveira-Moriyama L, Petrie A, Williams DR et al: The use of a color coded probability scale to interpret smell tests in suspected parkinsonism. Mov Disord 2009; 24: 1144-1153.

22 Scheltens $P$, Barkhof $F$, Leys $D$ et al: A semiquantative rating scale for the assessment of signal hyperintensities on magnetic resonance imaging. J Neurol Sci 1993; 114: 7-12.

$23 \mathrm{Wu}$ Y, Wu P: CD133 as a marker for cancer stems cells: progresses and concerns. Stem Cells Dev 2009; 18: 1127-1134.
24 Werner N, Nickenig G: Influence of cardiovascular risk factors on endothelial progenitor cells: limitations for therapy? Arterioscler Thromb Vasc Biol 2006; 26: 257-266.

25 Doeuvre L, Plawinski L, Toti $\mathrm{F}$ et al: Cell-derived microparticles: a new challenge in neuroscience. J Neurochem 2009; 110: 457-468.

26 Morel O, Toti F, Hugel B et al: Procoagulant microparticles: disrupting the vascular homeostasis equation? Arterioscler Thromb Vasc Biol 2006; 26: 2594-2604.

27 Chironi GN, Boulanger CM, Simon A et al: Endothelial microparticles in diseases. Cell Tissue Res 2009; 335: 143-151.

28 Sabatier F, Camoin-Jau L, Anfosso F et al: Circulating endothelial cells, microparticles and progenitors: key players towards the definition of vascular competence. J Cell Mol Med 2009; 13: 454-471.

29 Westerweel PE, Hoefer IE, Blankestijn PJ et al: End-stage renal disease causes an imbalance between endothelial and smooth muscle progenitor cells. Am J Physiol Renal Physiol 2007; 292: F1132-F1140.

30 Herbrig K, Pistrosch F, Foerster S et al: Endothelial progenitor cells in chronic renal insufficiency. Kidney Blood Press Res 2006; 29: 24-31.

31 Demir R, Seval Y, Huppertz B: Vasculogenesis and angiogenesis in the early human placenta. Acta Histochem 2007; 109: 257-265.

32 Urbich C, Dimmeler S: Endothelial progenitor cells functional characterization. Trends Cardiovasc Med 2004; 14: 318-322.

33 Zeppernick F, Ahmadi R, Campos B et al: Stem cell marker CD133 affects clinical outcome in glioma patients. Clin Cancer Res 2008; 14: 123-129.

34 Cantley LG: Adult stem cells in the repair of the injured renal tubule. Nat Clin Pract Nephrol 2005; 1: 22-32.

35 Minagar A, Jy W, Jimenez JJ et al: Elevated plasma endothelial microparticles in multiple sclerosis. Neurology 2001; 56: 1319-1324.

36 Knottnerus IL, Ten Cate H, Lodder J et al: Endothelial dysfunction in lacunar stroke: a systematic review. Cerebrovasc Dis 2009; 27: 519-526.

37 Asahara T, Masuda H, Takahashi T et al: Bone marrow origin of endothelial progenitor cells responsible for postnatal vasculogenesis in physiological and pathological neovascularization. Circ Res 1999; 85: 221-228.

38 Brown WR, Moody DM, Thore CR et al: Microvascular changes in the white mater in dementia. J Neurol Sci 2009; 283: 28-31.

39 Fargeas CA, Huttner WB, Corbeil D: Nomenclature of prominin-1 (CD133) splice variants - an update. Tissue Antigens 2007; 69: 602-606

40 Pleshkan VV, Vinogradova TV, Sverdlov ED: Methylation of the prominin 1 TATA-less main promoters and tissue specificity of their transcript content. Biochim Biophys Acta 2008; 1779: 599-605.

41 Hermann A, Maisel M, Liebau S et al: Mesodermal cell types induce neurogenesis from adult human hippocampal progenitor cells. J Neurochem 2006; 98: 629-640.

42 Pras $\mathrm{E}, \mathrm{Abu} \mathrm{A}$, Rotenstreich $\mathrm{Y}$ et al: Cone-rod dystrophy and a frameshift mutation in the PROM1 gene. Mol Vis 2009; 15: 1709-1716.

Supplementary Information accompanies the paper on European Journal of Human Genetics website (http://www.nature.com/ejhg) 